\title{
The Chemistry of Nearby Disks
}

\author{
Karin I. Öberg \\ Harvard-Smithsonian Center for Astrophysics, 60 Garden St, MS 16, Cambridge, MA 02138 \\ email: koberg@cfa.harvard.edu
}

\begin{abstract}
The gas and dust rich disks around young stars are the formation sites of planets. Observations of molecular trace species have great potential as probes of the disk structures and volatile compositions that together regulate planet formation. The disk around young star TW Hya has become a template for disk molecular studies due to a combination of proximity, a simple face-on geometry and richness in volatiles. It is unclear, however, how typical the chemistry of the TW disk is. In this proceeding, we review lessons learnt from exploring the TW Hya disk chemistry, focusing on the CO snowline, and on deuterium fractionation chemistry. We compare these results with new ALMA observations toward more distant, younger disks. We find that while all disks have some chemical structures in common, there are also substantial differences between the disks, which may be due to different initial conditions, structural or chemical evolutionary stages, or a combination of all three.
\end{abstract}

Keywords. astrochemistry, protoplanetary disks, ISM: molecules, radio lines: ISM

\section{Introduction}

Gas and dust-rich disks seem ubiquitous around young stars. The planet formation potential of these disks depends fundamentally on the disk dust and gas mass. Measuring disk masses is difficult, however. We are practically blind to the main constituent, cold $\mathrm{H}_{2}$ gas. Observations of dust continuum emission is a popular proxy, assuming a constant gas-to-dust conversion ratio. Disk demographic studies relying on such measurements suggest that the material needed to assemble a planetary system similar to our Solar System is frequently present around young stars (Andrews et al. 2013). Even around the smallest stars, there is more than sufficient mass in grains to form several Earthlike planets. This finding is in good agreement with exo-planet statistics, including the inferred high frequency of rocky planets around $\mathrm{M}$ dwarfs (Dressing \& Charbonneau 2015). Assuming a constant gas-to-dust ratio may be a poor approximation, however, due to a combination of dust growth into boulders that are hidden from view, decoupled dust and gas dynamics, and efficient gas photoevaporation from the disk atmosphere. CO line observations have been used as a more 'direct' tracer of the disk gas mass (Williams \& Bast 2014), but suffer from their own set of constraints, including a poorly constrained chemistry and therefore CO-to- $\mathrm{H}_{2}$ conversion factor under many disk conditions (Favre et al. 2013). Constraining this chemistry and therefore the utility of CO as a disk gas tracer should clearly be a priority for upcoming disk studies (Bergin et al. 2013). This statement can be generalized to other molecular tracers or important disk characteristics, such as temperature, density, and ionization profiles.

Understanding the chemical compositions of disks is also an end in itself. The compositions of planets depend fundamentally on the composition of the disk dust grains and disk volatiles they form from, and on how this composition varies with distance from the central star. Of especial importance for planet formation is the separation of volatiles into their gas and ice phases, which is set by the balance of adsorption ("freeze-out" of the gas phase onto solids), and desorption (back to the gas phase). We call the midplane 


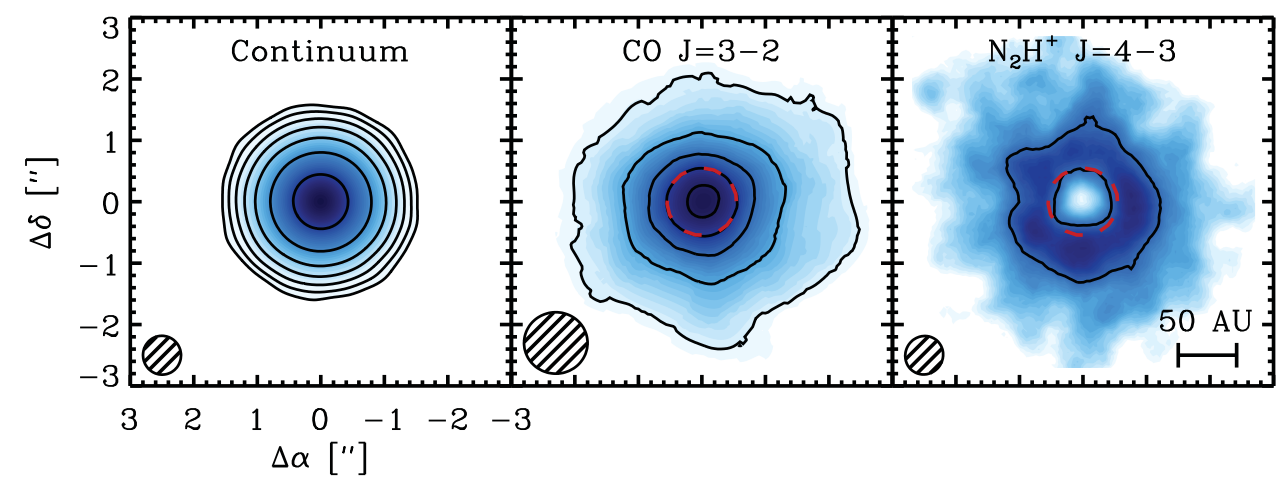

Figure 1. The dust continuum, $\mathrm{CO}$ and $\mathrm{N}_{2} \mathrm{H}^{+}$emission toward TW Hya. The red dashed circle marks the inferred $\mathrm{CO}$ snowline location. (reprinted with permission from Qi et al. 2013b).

radius where freeze-out overtakes desorption for a major volatile a 'snowline'. Because of an exponential dependence of desorption rates on temperature these transitions are expected to be sharp and may thus be properly referred to as 'lines' when only considering the midplane regions. Snowlines can enhance the planet formation efficiency through a combination of increased grain surface density, rapid particle growth due to cold-head effects, pressure traps and increased grain stickiness (Ciesla \& Cuzzi 2006, Johansen et al. 2007, Chiang \& Youdin 2010, Gunlach et al. 2011, Ros \& Johansen 2013). Snowline locations will also regulate the compositions of forming planets (Öberg et al. 2011) and planetesimals. In this proceeding we will only consider the CO snowline, simply because it occurs at a low temperature, i.e. far from the central star, and can thus be resolved by ALMA (§2).

A third aspect of planet formation is how Earth (and by extension, Earth-like planets and exoplanets) obtained its water. The Earth's oceans may have outgassed from the mantle, or may have been delivered by icy bodies bombarding the young Earth, or a combination of both. One approach to resolve this question is to compare the terrestrial water isotopic composition (level of deuterium enrichment) with other Solar System and interstellar water reservoirs. Using deuterium fractionation as a probe of the thermal history of terrestrial water (and other volatiles) presupposes a good understanding of the distribution and chemistry of deuterated molecules in the Solar Nebula. This chemistry is not well constrained in disks, however, and in $\S 3$ we discuss what observations of different deuterated species in the TW Hya disk and in younger disks can teach us about deuterium fractionation during planet formation.

\section{Disk Snowlines}

The locations of major snowlines depend on the volatile composition (e.g. whether most nitrogen is in $\mathrm{N}_{2}$ or $\mathrm{NH}_{3}$ ), a balance between freeze-out and thermal and non-thermal desorption rates at different disk locations (set by a combination of density, temperature and radiation fields), and disk dynamics (Öberg et al. 2011). Exact snowline locations are therefore difficult to predict from theory, and observational constraints are key to constrain planet formation models. The $\mathrm{CO}$ snowline is expected at the disk radius where the temperature drops below $\sim 20 \mathrm{~K}$. Around T Tauri and Herbig Ae disks this threshold is crossed at $\sim 20-150$ AU.

Extractions of $\mathrm{CO}$ snowline locations using $\mathrm{CO}$ observations is challenging because of the presence of large amounts of CO in the disk atmosphere (Qi et al. 2011). An 
alternative approach is to exploit chemical effects regulated by $\mathrm{CO}$ freeze-out to constrain the location of the $\mathrm{CO}$ snow line. Three proposed $\mathrm{CO}$ snow line tracers are $\mathrm{N}_{2} \mathrm{H}^{+}, \mathrm{H}_{2} \mathrm{CO}$, and $\mathrm{DCO}^{+}$(Qi et al. 2013, Mathews et al. 2013). $\mathrm{N}_{2} \mathrm{H}^{+}$is expected to be a robust tracer of $\mathrm{CO}$ freeze-out because the presence of gas phase $\mathrm{CO}$ slows down $\mathrm{N}_{2} \mathrm{H}^{+}$formation and speeds up $\mathrm{N}_{2} \mathrm{H}^{+}$destruction. The predicted anti-correlation between gas-phase $\mathrm{CO}$ and $\mathrm{N}_{2} \mathrm{H}^{+}$is observed in pre-stellar and protostellar environments (e.g. Caselli et al. 1999, Bergin et al. 2002, Jørgensen et al. 2004). Qi et al. (2013b) imaged $\mathrm{N}_{2} \mathrm{H}^{+}$emission with the Atacama Large sub-Millimeter Array (ALMA) toward the disk around T Tauri star TW Hya and found a large ring with an inner edge at $30 \mathrm{AU}$, tracing the CO snowline (Fig. 1). The determined CO snowline location corresponds to a CO freeze-out temperature of $17 \mathrm{~K}$, using a detailed disk density and temperature structure. This is a few degrees lower than expected, which may reflect a combination of model uncertainties and excess CO depletion due to chemical conversion of $\mathrm{CO}$ into other species in this old disk.

With ALMA similar measurements using $\mathrm{N}_{2} \mathrm{H}^{+}$are possible toward samples of disks. So far only one such additional measurement exists. $\mathrm{N}_{2} \mathrm{H}^{+}$observations with ALMA toward HD 163296 reveal a similar ring-like structure as previously observed toward TW Hya, but the inferred CO snowline location is substantially (10s of AU) closer to the star than expected from the inferred CO freeze-out temperature in the TW Hya disk. The HD 163296 CO snowline measurement corresponds to a CO freeze-out temperature of $\sim 25 \mathrm{~K}, 8 \mathrm{~K}$ higher than in the TW Hya disk (C. Qi, private communication). Such a high freeze-out temperature may be explained by dynamics (moving the snowline inwards due to drift of solids) or a water-rich ice morphology. CO is known to bind stronger to water ice compared to other $\mathrm{CO}$ molecules and even small amounts of water forming or freezing out with the $\mathrm{CO}$ ice could increase the $\mathrm{CO}$ freeze-out temperature by several degrees (Collings et al. 2004).

In summary: there are two measurements of $\mathrm{CO}$ snowline locations and the results are two substantially different $\mathrm{CO}$ freeze-out temperatures. The causes for these differences are unclear, though there are several candidates, including disk dynamics and ice chemistry. This result entails that we cannot currently use theory to predict the CO freeze-out temperature or the location of the CO snowline in a disk, including the Solar Nebula. There is a clear need for a survey of CO snowline locations toward disks to 1 . constrain how CO freeze-out depends on disk structure and evolution, and 2. establish the 'typical' $\mathrm{CO}$ freeze-out temperature during planet formation.

\section{Deuterium Fractionation}

Deuterium fractionation is commonly used to infer (thermal) properties about volatile formation locations. Molecules become enriched in deuterium due to small differences in zero-point energies between deuterated and non-deuterated molecules, which results in preferential formation of deuterated molecules at low temperatures. Based on this understanding, the high $\mathrm{D} / \mathrm{H}$ ratio in terrestrial $\mathrm{H}_{2} \mathrm{O}$ has been used to infer delivery of water from asteroids and comets, which both assembled in the colder, outer Solar System (Hartogh et al. 2011). The generally high D/H ratio in Solar System water has further been used to infer a cold pre-Solar origin of most Solar System $\mathrm{H}_{2} \mathrm{O}$ (Cleeves et al. 2014).

So far only two deuterated molecules have been detected in disks, $\mathrm{DCO}^{+}$and $\mathrm{DCN}$ (van Dishoeck et al. 2003, Qi et al. 2008). Figure 2 shows Submillimeter Array (SMA) observations of $\mathrm{DCO}^{+}$in the TW Hya disk. The $\mathrm{DCO}^{+}$emission presents a low SNR ring. When compared with $\mathrm{HCO}^{+}$toward $\mathrm{TW}$ Hya, this ring implies an increasing level of deuterium fractionation with disk radius. This is in agreement with theoretical 


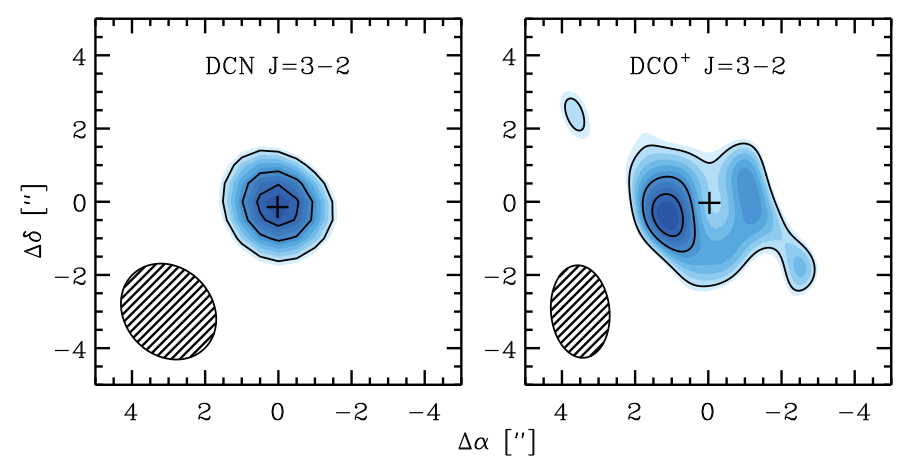

Figure 2. Integrated images of the TW Hya disk in (left:) DCN J=3-2 emission (ALMA science verification) and (right:) $\mathrm{DCO}^{+} \mathrm{J}=3-2$ emission (SMA data from Qi et al. (2008)). The contour levels are at $50 \%, 75 \%$ and $90 \%$ of the peak value. The cross marks the peak of the continuum emission, which locates the position of the central star. Reprinted with permission from Öberg et al. (2012).

expectations, since the temperature in the disk midplane decreases with increasing disk radius. By contrast, DCN emission presents a centrally peaked feature toward the TW Hya disk (Fig. 2). This suggests that deuterium fractionation in HCN occurs at higher temperatures compared to $\mathrm{HCO}^{+}$. There are clearly multiple pathways to deuterium fractionation in disks, which operate in different temperature regimes (Öberg et al. 2012). This already complicates the use of deuterium fractionation as a thermal history probe.

The picture is complicated further by recent observations of $\mathrm{DCO}^{+}$and $\mathrm{DCN}$ toward a small sample of disks with ALMA. Figure 3 shows the different $\mathrm{DCO}^{+}$and $\mathrm{DCN} 3-$ 2 emission morphologies toward three disks around T Tauri stars AS 209, V 4046 Sgr and IM Lup. These disks were observed during 2014 and 2015 with the Atacama Large Millimeter/Submillimeter Array (ALMA; project code 2013.1.00226) with 30 antennas, spanning baselines of $15-650 \mathrm{~m}$. The total on source integration time was $\sim 20$ minutes. The visibility data were calibrated by ALMA staff. Each individual SPW was further phase and amplitude self-calibrated in CASA 4.2.2. In each SPW, the continuum was subtracted using line-free channels. The fully calibrated visibilities were Fourier inverted and CLEANed.

Similarly to the TW Hya disk, the $\mathrm{DCO}^{+}$emission maps always present a hole or depressions toward the warm disk center. In contrast to the previous TW Hya observations, the DCN emission maps also present central depressions toward all three disks. This may simply be an effect of the increased spatial resolution employed for these observations, however. A second similarity between TW Hya and the disk sample is that the DCN emission appears to be more compact than the $\mathrm{DCO}^{+}$emission, suggesting that cold deuterium fractionation chemistry is generally more important for $\mathrm{DCO}^{+}$production compared to DCN. These structural similarities between TW Hya and the younger disk sample in Fig. 3 are accompanied by several important differences, however.

Figure 3 shows the relative sizes of the $\mathrm{DCO}^{+}$and $\mathrm{DCN}$ central emission depressions vary between different disks. Toward AS 209, the radius of the central $\mathrm{DCO}^{+}$hole is smaller than the DCN hole radius. The opposite is true for the relative hole sizes of $\mathrm{DCO}^{+}$ and DCN in the transition disk around V4046 Sgr, which also presents the largest $\mathrm{DCO}^{+}$ hole in the sample. IM Lup presents the most surprising morphology, a set of concentric rings in $\mathrm{DCO}^{+}$emission (Öberg et al. 2015, submitted to ApJ). DCN is barely detected in this source. Together with TW Hya, this small disk sample reveals that there is no simple relationship between disk radius and the deuterium pathways that results in deuterium 




Figure 3. $\mathrm{DCO}^{+}$and DCN emission toward three protoplanetary disks. The left panels show the integrated emission maps of $\mathrm{DCO}^{+}$and $\mathrm{DCN}$ with the color scale marking the flux density in $\mathrm{mJy} \mathrm{km} / \mathrm{s} \mathrm{beam}^{-1}$ (the beam is shown in the bottom left of each panel). The right panels show the corresponding deprojected and azimuthally averaged radial profiles.

enrichments of either $\mathrm{HCO}^{+}$or $\mathrm{HCN}$. In particular DCN does not always extend further in toward the central star compared to $\mathrm{DCO}^{+}$. In summary: even when only considering two molecules, the deuterium enrichment pattern observed toward TW Hya does not appear to be universal in protoplanetary disks.

\section{Conclusions}

TW Hya has been and will continue to be an important starting point when studying disk chemistry (see, e.g., Kastner et al. 1997, Thi et al. 2004, Qi et al. 2013b). Its proximity, well-established disk density and temperature structure and rich molecular inventory makes it ideal to develop new chemical probes. The chemistry of the TW Hya disk may be far from typical, however. The disk around TW Hya is old compared to most other gas-rich circumstellar disks, and disk chemistry is time dependent. In this proceeding we compared the observed chemistry in the TW Hya disk with other, younger disks focusing on two key processes: the chemical imprint of $\mathrm{CO}$ freeze-out, and deuterium fractionation. In both cases we found several similarities between the TW Hya disk and the other targets, but also substantial differences. For example, the $\mathrm{CO}$ snowline locations in the TW Hya and HD 163296 disks do not seem to correspond to the same CO freeze-out temperature, and the more centrally peaked DCN emission compared to $\mathrm{DCO}^{+}$observed toward TW Hya is not reproduced toward all other disks. Whether these differences trace different initial conditions, present differences in disk temperature, density and radiation structures, or different chemical evolutionary stages is currently unclear. Larger surveys of disk chemistry should be able to answer some of these questions, when combining the survey results with detailed disk density, temperature and radiation structures, theory 
and laboratory work. Only then can we expect to establish the 'typical' chemistry present during planet formation, and further, the origins of the present-day Solar System chemical composition.

This paper makes use of the following ALMA data: ADS/JAO.ALMA\#2013.1.00226.S. ALMA is a partnership of ESO (representing its member states), NSF (USA) and NINS (Japan), together with NRC (Canada) and NSC and ASIAA (Taiwan), in cooperation with the Republic of Chile. The Joint ALMA Observatory is operated by ESO, AUI/NRAO and NAOJ. The National Radio Astronomy Observatory is a facility of the National Science Foundation operated under cooperative agreement by Associated Universities, Inc. KIÖ also acknowledges funding from the Alfred P. Sloan Foundation, and the Packard Foundation.

\section{References}

Andrews, S. M., Rosenfeld, K. A., Kraus, A. L., \& Wilner, D. J. 2013, ApJ, 771, 129

Bergin, E. A., Alves, J., Huard, T., \& Lada, C. J. 2002, ApJL, 570, L101

Bergin, E. A., Cleeves, L. I., Gorti, U., et al. 2013, Nature, 493, 644

Caselli, P., Walmsley, C. M., Tafalla, M., Dore, L., \& Myers, P. C. 1999, ApJL, 523, L165

Chiang, E. \& Youdin, A. N. 2010, Annual Review of Earth and Planetary Sciences, 38, 493

Ciesla, F. J. \& Cuzzi, J. N. 2006, Icarus, 181, 178

Cleeves, L. I., Bergin, E. A., Alexander, C. M. O., et al. 2014, Science, 345, 1590

Collings, M. P., Anderson, M. A., Chen, R., et al. 2004, MNRAS, 354, 1133

Dressing, C. D. \& Charbonneau, D. 2015, ArXiv e-prints

Favre, C., Cleeves, L. I., Bergin, E. A., Qi, C., \& Blake, G. A. 2013, ApJL, 776, L38

Gundlach, B., Kilias, S., Beitz, E., \& Blum, J. 2011, Icarus, 214, 717

Hartogh, P., Lis, D. C., Bockelée-Morvan, D., et al. 2011, Nature, 478, 218

Johansen, A., Oishi, J. S., Low, M.-M. M., et al. 2007, Nature, 448, 1022

Jørgensen, J. K. 2004, A\& A, 424, 589

Kastner, J. H., Zuckerman, B., Weintraub, D. A., \& Forveille, T. 1997, Science, 277, 67

Mathews, G. S., Klaassen, P. D., Juhász, A., et al. 2013, A\&\&A, 557, A132

Öberg, K. I., Murray-Clay, R., \& Bergin, E. A. 2011a, ApJL, 743, L16

Öberg, K. I., Qi, C., Wilner, D. J., \& Hogerheijde, M. R. 2012, ApJ, 749, 162

Qi, C., D'Alessio, P., Öberg, K. I., et al. 2011, ApJ, 740, 84

Qi, C., Öberg, K. I., \& Wilner, D. J. 2013a, ApJ, 765, 34

Qi, C., Öberg, K. I., Wilner, D. J., et al. 2013b, Science, 341, 630

Qi, C., Wilner, D. J., Aikawa, Y., Blake, G. A., \& Hogerheijde, M. R. 2008, ApJ, 681, 1396

Ros, K. \& Johansen, A. 2013, A\&A A, 552, A137

Thi, W., van Zadelhoff, G., \& van Dishoeck, E. F. 2004, ApJ, 425, 955

van Dishoeck, E. F., Thi, W., \& van Zadelhoff, G. 2003, $A \& A$, 400, L1

Williams, J. P. \& Best, W. M. J. 2014, ApJ, 788, 59 